\title{
The ARID1B spectrum in 143 patients: from nonsyndromic intellectual disability to Coffin-Siris syndrome
}

\author{
A full list of authors and affiliations appears at the end of the paper.
}

\begin{abstract}
Purpose: Pathogenic variants in ARID1B are one of the most frequent causes of intellectual disability (ID) as determined by large-scale exome sequencing studies. Most studies published thus far describe clinically diagnosed Coffin-Siris patients (ARID1BCSS) and it is unclear whether these data are representative for patients identified through sequencing of unbiased ID cohorts (ARID1B-ID). We therefore sought to determine genotypic and phenotypic differences between ARID1B-ID and ARID1B-CSS. In parallel, we investigated the effect of different methods of phenotype reporting.
\end{abstract}

Methods: Clinicians entered clinical data in an extensive webbased survey.

Results: 79 ARID1B-CSS and 64 ARID1B-ID patients were included. CSS-associated dysmorphic features, such as thick eyebrows, long eyelashes, thick alae nasi, long and/or broad philtrum, small nails and small or absent fifth distal phalanx and hypertrichosis, were observed significantly more often $(p<0.001)$ in ARID1B-CSS patients. No other significant differences were identified.

Conclusion: There are only minor differences between ARID1BID and ARID1B-CSS patients. ARID1B-related disorders seem to consist of a spectrum, and patients should be managed similarly. We demonstrated that data collection methods without an explicit option to report the absence of a feature (such as most Human Phenotype Ontology-based methods) tended to underestimate gene-related features.

Genetics in Medicine (2019) 21:1295-1307; https://doi.org/10.1038/s41436018-0330-Z

Keywords: ARID1B; Coffin-Siris syndrome; intellectual disability; bias

\section{INTRODUCTION}

The overall prevalence of intellectual disability (ID) has been estimated at around $1 \%$. Given the increasing number of genes involved in ID, exome sequencing is becoming the first method of choice to identify the underlying genetic cause in patients with ID. ${ }^{1}$ This unbiased approach detects clearly pathogenic variants in patients without the typically associated phenotype, indicating that variability in expression is higher than previously thought, confirming the existence of ascertainment bias. This bias may be mitigated by establishing the frequency of cardinal features in patients diagnosed through sequencing of unselected populations, although selection criteria for patients who undergo sequencing still cause a degree of bias. However, studies in unbiased populations suffer from another, less appreciated bias: they may underestimate the frequency of cardinal features because of the way that data are collected in large research studies. We would like to coin this phenomenon "phenotype underreporting bias": typically, a busy clinician is requested to supply several Human Phenotype Ontology (HPO) terms, and there is no guarantee that all features have been assessed or that all the clinical information is reported by the clinician.
Consequently, it is not possible to make a distinction between the absence of a feature and unknown status, especially when specific diagnostic procedures are required to assess a feature (e.g., a magnetic resonance image [MRI] scan for agenesis of the corpus callosum [ACC]).

The ARID1B phenotype represents a good case study to investigate these biases, because the associated phenotypes range from clearly recognizable Coffin-Siris syndrome (ARID1B-CSS) to less specific ID $^{2,3}$ (ARID1B-ID). ARID1B is by far the most frequently mutated gene (51-75\%) (refs. ${ }^{4-6}$ ) in Coffin Siris syndrome (CSS) (OMIM 135900) and large-scale exome sequencing studies invariably find that pathogenic variants in ARID1B are among the most frequently identified causes in unspecified ID cohorts (usually around 1\%) (refs. ${ }^{1,2}$ ).

CSS is characterized by "developmental or cognitive delay, hypotonia, sparse scalp hair, distinctive facial features, aplasia or hypoplasia of the distal phalanx or nail of the fifth and additional digits, and hypertrichosis." 7 Approximately 70 ARID1B-CSS patients ${ }^{4-6,8-12}$ have been described. Roughly 30 ARID1B-ID patients have been described in some detail ${ }^{2,13-24}$ and an additional 23 patients with pathogenic variants in ARID1B were identified in genome-wide research studies ${ }^{1,24}$ 
where detailed clinical information is generally unavailable. Data on the frequency of typical CSS features in the ARID1BID population are lacking because a substantial number of these patients were published before the link between ARID1B and CSS was known. For example, in Hoyer et al. ${ }^{2}$ ACC was not specifically reported, although this is now known as a frequent feature in ARID1B-CSS. ${ }^{11}$ Therefore, the precise prevalence of these features cannot be estimated. The availability of unbiased information is crucial now that exome sequencing is being performed increasingly in neonatal and prenatal settings, and a reliable prognosis can only be given based on unbiased data.

The first aim of this study was therefore to overcome ascertainment and phenotype underreporting biases, by acquiring detailed clinical data of a large cohort of ARID1B-ID patients. The second aim was to determine whether the frequencies of features differ between ARID1BCSS and ARID1B-ID patients, as would be expected at least for typical CSS features.

\section{MATERIALS AND METHODS}

Patient ascertainment

We developed a web-based survey (www.aridlbgene.com) based on previously reported features of ARID1B patients. This website is part of the Human Disease Genes website series (HDG), a collection of websites aimed at informing professionals about genes and copy-number variations and their clinical consequences (http://humandiseasegenes.com/). The survey was open to all clinicians of patients with pathogenic variants in ARIDIB. Data were contributed by pediatric neurologists, pediatricians, and in most instances by clinical geneticists. Some clinicians contacted us, others were approached based on publications, conference presentations, submissions to databases like DECIPHER, or through large laboratories. We also included patients from our previous studies ${ }^{4-6,9,11}$ and those referred to our national CSS expertise center in Leiden, The Netherlands.

The institutional review board of the Leiden University Medical Center, Leiden, The Netherlands provided an approval waiver for this study.

\section{Data assessment}

When only partial variant data was given, the remaining information was recovered using Alamut version 2.6.0. When the standard deviation score (SDS) was not reported, but raw data on weight, height, or occipital-frontal circumference (OFC) was available, the SDS was determined using published growth charts. ${ }^{25}$

After initial analyses we recontacted contributing clinicians by email to inquire about features frequently reported in open-ended questions.

We used a nominal $p$ value of 0.05 as a cut-off for significance. However, given that we assessed 90 features, multiplicity correction by Bonferroni suggests that $p$ values above $0.0006(0.05 / 90)$ should be treated with care. All analyses were executed using SPSS version 23. R version 3.4.1 was used to create graphs, including the survival package (version 2.41-3).

\section{RESULTS}

Data from 143 individuals with pathogenic variants in $A R I D 1 B$ were included in the database. We received additional data regarding features recurrently indicated in our open-ended questions (Table 1 features marked with " + +", Supplementary Table S4) of 95 patients. Supplementary Figure S1 displays facial photographs and hands or feet from two ARID1B-ID and two ARID1B-CSS patients. Parents provided consent for publication of these pictures.

\section{Genotype}

Figure 1 and Supplementary Table S1 provide an overview of the submitted pathogenic variants. Sixty-two patients were previously reported in literature. ${ }^{4-6,9,19,26}$ Pathogenic variants were apparently de novo in all cases where parents could be tested $(107 / 107)$. In two sisters the same pathogenic variant was found, while paternal DNA could not be obtained. Most pathogenic variants were frameshift or nonsense $(n=118), 18$ were deletions involving multiple or all exons, and 7 involved canonical splice sites. One patient with a missense variant was initially submitted but later retracted when the variant turned out to be inherited from the unaffected father.

\section{Phenotype}

Patients' characteristics are summarized in Table $\mathbf{1}$ and Supplementary Table S2. Of the 143 individuals, 69 (48.3\%) were female and the age at follow-up varied between 0 and 51 years with a median of 10 years. ARID1B-ID patients were less likely to be female than ARID1B-CSS patients (38 vs. $57 \%, p=0.028$ ). Two individuals have died, one of brain swelling potentially due to low-grade brain stem encephalitis that led to cardiorespiratory arrest at the age of 9 years, and one suddenly after the age of 24 years of unknown cause.

\section{Diagnosis before genetic testing}

Seventy-nine ARID1B-CSS patients were included. Most (91.5\%) of the 64 ARID1B-ID patients were retrospectively classified to fit the CSS spectrum by the referring clinician. An overview of the clinical diagnoses prior to identification of the pathogenic variant can be found in Supplementary Table S3. No statistically significant differences in phenotypic features were found between patients who were retrospectively classified to fit the CSS spectrum by their referring clinicians $(n=54)$ and those who were not $(n=5)$.

\section{Comparison of the ARID1B-ID and ARID1B-CSS groups}

As expected, ARID1B-CSS patients more frequently displayed features associated with CSS than ARID1B-ID patients, including thick eyebrows, long eyelashes, thick alae nasi, long and/or broad philtrum, small nails and small or absent fifth distal phalanx, and hypertrichosis $(p<0.0001-0.001$, Table 1).

ARID1B-ID patients appeared to have a higher prevalence of myopia $(p=0.020)$, cryptorchidism $(p=0.023)$, constipation $(p=0.002)$, sleep apnea $(p=0.026)$, hyperactivity $(p=0.026)$, and high pain threshold $(p=0.044)$, although these differences 
Table 1 Clinical characteristics of ARID1B patients

\begin{tabular}{|c|c|c|c|c|c|c|c|c|}
\hline \multirow[b]{2}{*}{ Clinical features $^{d}$} & \multirow{2}{*}{$\begin{array}{l}\text { Total } \\
n=143\end{array}$} & \multirow[b]{2}{*}{$\%$} & \multicolumn{2}{|c|}{ ARID1B-CSS } & \multicolumn{2}{|c|}{ ARID1B-ID } & \multirow[b]{2}{*}{$p$ value } & \multirow[b]{2}{*}{ Test } \\
\hline & & & $n=79$ & $\%$ & $n=64$ & $\%$ & & \\
\hline Sex (female) & 143 & 48.3 & 79 & 57.0 & 64 & 37.5 & 0.028 & a \\
\hline \multicolumn{9}{|l|}{ Growth parameters \& development } \\
\hline Gestational age, weeks (mean; SD) & 133 & $39.0 ; 2.1$ & 75 & $39.1 ; 2.0$ & 58 & $38.9 ; 2.4$ & 0.879 & b \\
\hline Birthweight (<-2 SDS) & 129 & 5.4 & 74 & 6.8 & 55 & 3.6 & 0.506 & c \\
\hline Height at birth $(<-2$ SDS) & 43 & 9.3 & 27 & 11.1 & 16 & 6.3 & 0.660 & c \\
\hline OFC at birth $(<-2$ SDS $)$ & 51 & 3.9 & 35 & 2.9 & 16 & 6.3 & 0.232 & c \\
\hline Age last measurements, years (median; min-max) & 143 & $10 ; 0-51$ & 79 & $10 ; 0-36$ & 64 & $9 ; 0.5-51$ & 0.682 & b \\
\hline Weight (<-2 SDS) & 92 & 6.5 & 46 & 8.7 & 46 & 4.3 & 0.571 & c \\
\hline Height (<-2 SDS) & 122 & 30.3 & 70 & 37.1 & 52 & 21.2 & 0.177 & c \\
\hline OFC (<-2 SDS) & 105 & 2.9 & 63 & 3.2 & 42 & 2.4 & 0.670 & c \\
\hline Motor skills gross, delayed & 103 & 99.0 & 46 & 97.8 & 57 & 100.0 & 0.447 & c \\
\hline Motor skills fine, delayed & 100 & 95.0 & 44 & 97.7 & 56 & 92.9 & 0.381 & c \\
\hline Speech, delayed & 131 & 65.6 & 75 & 68.0 & 56 & 62.5 & 0.106 & c \\
\hline Obstructive sleep apnea ${ }^{\mathrm{e}}$ & 71 & 8.5 & 34 & 0.0 & 37 & 16.2 & 0.026 & c \\
\hline Laryngomalacia $^{\mathrm{e}}$ & 90 & 19.8 & 47 & 17.0 & 44 & 22.7 & 0.466 & a \\
\hline Feeding difficulties & 121 & 69.4 & 62 & 62.9 & 59 & 76.3 & 0.111 & a \\
\hline Start of feeding difficulties & 71 & & 34 & & 37 & & 0.345 & c \\
\hline Birth & & 76.1 & & 76.5 & & 75.7 & & \\
\hline Before 6 months & & 16.9 & & 20.6 & & 13.5 & & \\
\hline After 6 months & & 7.0 & & 2.9 & & 10.8 & & \\
\hline Duration of feeding problems & 58 & & 23 & & 35 & & 0.639 & c \\
\hline Brief & & 46.6 & & 39.1 & & 51.4 & & \\
\hline Several years & & 6.9 & & 8.7 & & 5.7 & & \\
\hline Ongoing & & 46.6 & & 52.2 & & 42.9 & & \\
\hline Tube feeding & 65 & 16.9 & 22 & 13.6 & 43 & 18.6 & 0.409 & c \\
\hline $0-6$ months & & 10.8 & & 4.5 & & 14.0 & & \\
\hline 6-12 months & & 3.1 & & 4.5 & & 2.3 & & \\
\hline $1-3$ years & & 1.5 & & 0.0 & & 2.3 & & \\
\hline Recurrent infections & 75 & 57.3 & 30 & 63.3 & 45 & 53.3 & 0.391 & a \\
\hline Upper airway tract & & 17.3 & & 10.0 & & 22.2 & & \\
\hline Lower airway tract & & 2.7 & & 0.0 & & 4.4 & & \\
\hline ENT infections & & 12.0 & & 10.0 & & 13.3 & & \\
\hline Otitis media & & 14.7 & & 13.3 & & 15.6 & & \\
\hline Urinary tract & & 2.7 & & 3.3 & & 2.2 & & \\
\hline \multicolumn{9}{|l|}{ Neurological features } \\
\hline Intellectual disability & 127 & 99.2 & 70 & 98.6 & 57 & 100.0 & 0.015 & c \\
\hline Normal-mild & & 3.1 & & 1.4 & & 5.3 & & \\
\hline Mild & & 28.3 & & 38.6 & & 15.8 & & \\
\hline Mild-moderate & & 15.7 & & 8.6 & & 24.6 & & \\
\hline Moderate & & 22.0 & & 22.9 & & 21.1 & & \\
\hline Moderate-severe & & 16.5 & & 17.1 & & 15.8 & & \\
\hline Severe & & 13.4 & & 10.0 & & 17.5 & & \\
\hline Hypotonia & 116 & 81.0 & 71 & 80.3 & 45 & 82.2 & 0.795 & a \\
\hline Seizures & 142 & 27.5 & 78 & 28.2 & 64 & 26.6 & 0.880 & c \\
\hline No seizures, but abnormal EEG & & 5.6 & & 6.4 & & 4.7 & & \\
\hline Seizure frequency & 18 & & 9 & & 9 & & 0.671 & c \\
\hline Once & & 27.8 & & 11.1 & & 44.4 & & \\
\hline Less than once a year & & 11.1 & & 22.2 & & 0.0 & & \\
\hline Once a year & & 33.3 & & 44.4 & & 22.2 & & \\
\hline Once a month & & 11.1 & & 11.1 & & 11.1 & & \\
\hline
\end{tabular}


Table 1 continued

\begin{tabular}{|c|c|c|c|c|c|c|c|c|}
\hline \multirow[b]{2}{*}{ Clinical features ${ }^{d}$} & \multirow{2}{*}{$\begin{array}{l}\text { Total } \\
n=143\end{array}$} & \multirow[b]{2}{*}{$\%$} & \multicolumn{2}{|c|}{ ARID1B-CSS } & \multicolumn{2}{|c|}{ ARID1B-ID } & \multirow[b]{2}{*}{$p$ value } & \multirow[b]{2}{*}{ Test } \\
\hline & & & $n=79$ & $\%$ & $n=64$ & $\%$ & & \\
\hline $1 / 2$ a month & & 5.6 & & 0.0 & & 11.1 & & \\
\hline$\geq 2$ per month & & 5.6 & & 0.0 & & 11.1 & & \\
\hline Agenesis of the corpus callosum & 101 & 28.7 & 62 & 29.0 & 39 & 28.2 & 0.344 & c \\
\hline Partial/hypoplasia & & 13.9 & & 17.7 & & 7.7 & & \\
\hline Neuroradiology & 47 & 87.2 & 17 & 94.1 & 30 & 83.3 & 0.305 & a \\
\hline Delayed myelination & & 17.0 & & 11.8 & & 20.0 & & \\
\hline Mega cisterna magna & & 14.9 & & 23.5 & & 10.0 & & \\
\hline Colpocephaly & & 10.6 & & 11.8 & & 10.0 & & \\
\hline Hypoplasia & & 4.3 & & 0.0 & & 6.7 & & \\
\hline Enlarged Virchow-Robin spaces & & 4.3 & & 5.9 & & 3.3 & & \\
\hline \multicolumn{9}{|l|}{ Vision and hearing impairments } \\
\hline Vision impaired & 109 & 48.6 & 62 & 45.2 & 47 & 53.2 & 0.406 & a \\
\hline Vision problems & 68 & 70.6 & 33 & 78.8 & 35 & 62.9 & 0.320 & c \\
\hline Astigmatism & & 16.2 & & 24.2 & & 8.6 & & \\
\hline Strabismus & & 30.9 & & 36.4 & & 25.7 & & \\
\hline Optic nerve hypoplasia & & 2.9 & & 6.1 & & 0.0 & & \\
\hline Nystagmus & & 8.8 & & 6.1 & & 11.4 & & \\
\hline Refraction error & & 10.3 & & 9.1 & & 11.4 & & \\
\hline Myopia & 102 & 27.5 & 59 & 18.6 & 43 & 39.5 & 0.020 & a \\
\hline Hypermetropia & 50 & 18.0 & 21 & 28.6 & 29 & 10.3 & 0.140 & c \\
\hline Abnormal eye exam & 40 & 17.5 & 15 & 6.7 & 25 & 24.0 & 0.224 & c \\
\hline Hearing loss & 122 & 22.1 & 71 & 18.3 & 51 & 27.5 & 0.157 & a \\
\hline Hearing loss, conductive & & 6.6 & & 1.4 & & 13.7 & & \\
\hline Hearing loss, bilateral & & 11.5 & & 8.5 & & 15.7 & & \\
\hline Hearing loss, unilateral & & 4.9 & & 5.6 & & 3.9 & & \\
\hline Eartubes & & 4.9 & & 4.2 & & 5.9 & & \\
\hline Start hearing problems, congenital & 11 & 63.6 & 3 & 66.7 & 8 & 62.5 & 0.109 & c \\
\hline Hearing aid & 5 & 80.0 & 2 & 100.0 & 3 & 66.7 & 0.665 & c \\
\hline \multicolumn{9}{|l|}{ Dysmorphic features } \\
\hline Coarse face & 121 & 81.8 & 62 & 90.3 & 59 & 72.9 & 0.013 & a \\
\hline Hairline (low anterior and/or posterior) & 91 & 69.2 & 45 & 75.6 & 46 & 63.0 & 0.196 & a \\
\hline Scalp hair, abnormal & 129 & 79.1 & 78 & 83.3 & 51 & 72.5 & 0.141 & a \\
\hline Sparse & & 58.1 & & 62.8 & & 51.0 & & \\
\hline Forehead (broad or narrow) & 95 & 42.1 & 49 & 28.6 & 46 & 56.5 & 0.000 & c \\
\hline Broad & & 22.1 & & 6.1 & & 39.1 & & \\
\hline Narrow & & 20.0 & & 22.4 & & 17.4 & & \\
\hline Eyelashes, long & 131 & 63.4 & 79 & 75.9 & 52 & 44.2 & 0.000 & a \\
\hline Eyebrows, thick & 134 & 81.3 & 78 & 91.0 & 56 & 67.9 & 0.001 & a \\
\hline Ptosis & 133 & 20.3 & 77 & 20.8 & 56 & 19.6 & 0.872 & a \\
\hline Tear duct nonfunctioning or absent & 93 & 15.1 & 54 & 16.7 & 39 & 12.8 & 0.609 & a \\
\hline Nasal bridge, abnormal & 100 & 61.0 & 59 & 62.7 & 41 & 58.5 & 0.050 & a \\
\hline Wide & & 34.0 & & 40.7 & & 24.4 & & \\
\hline Flat & & 21.0 & & 20.3 & & 22.0 & & \\
\hline Broad & & 12.0 & & 5.1 & & 22.0 & & \\
\hline Nasal tip, abnormal & 129 & & 76 & & 53 & & 0.002 & a \\
\hline Broad & & 58.1 & & 61.8 & & 52.8 & & \\
\hline Upturned (anteverted nares) & & 29.5 & & 39.5 & & 15.1 & & \\
\hline Nose, abnormal & 83 & 47.0 & 43 & 53.5 & 40 & 40.0 & 0.022 & a \\
\hline Short & & 26.5 & & 39.5 & & 12.5 & & \\
\hline Long & & 20.5 & & 14.0 & & 27.5 & & \\
\hline
\end{tabular}


Table 1 continued

\begin{tabular}{|c|c|c|c|c|c|c|c|c|}
\hline \multirow[b]{2}{*}{ Clinical features $^{d}$} & \multirow{2}{*}{$\begin{array}{l}\text { Total } \\
n=143\end{array}$} & \multirow[b]{2}{*}{$\%$} & \multicolumn{2}{|c|}{ ARID1B-CSS } & \multicolumn{2}{|c|}{ ARID1B-ID } & \multirow[b]{2}{*}{$p$ value } & \multirow[b]{2}{*}{ Test } \\
\hline & & & $n=79$ & $\%$ & $n=64$ & $\%$ & & \\
\hline Alae nasi, thick & 107 & 55.1 & 69 & 66.7 & 38 & 34.2 & 0.001 & a \\
\hline Nasal base, broad & 88 & 48.9 & 48 & 43.8 & 40 & 55.0 & 0.392 & a \\
\hline Philtrum, abnormal & 109 & 78.9 & 72 & 86.1 & 37 & 64.9 & 0.001 & a \\
\hline Short & & 24.8 & & 29.2 & & 16.2 & & \\
\hline Long & & 44.0 & & 48.6 & & 35.1 & & \\
\hline Broad & & 34.9 & & 44.4 & & 16.2 & & \\
\hline Mouth, large & 131 & 68.7 & 75 & 76.0 & 56 & 58.9 & 0.037 & a \\
\hline Upper vermillion, abnormal & 127 & 56.7 & 75 & 60.0 & 52 & 51.9 & 0.366 & a \\
\hline Thin & & 35.4 & & 45.3 & & 21.2 & & \\
\hline Thick & & 21.3 & & 14.7 & & 30.8 & & \\
\hline Lower vermillion, thick & 125 & 69.6 & 76 & 78.9 & 49 & 55.1 & 0.005 & a \\
\hline Lower lip, drooping & 71 & 56.3 & 30 & 76.7 & 41 & 41.5 & 0.004 & a \\
\hline Cleft palate/submucous cleft & 90 & 6.7 & 35 & 14.3 & 55 & 1.8 & 0.031 & a \\
\hline Cleft palate & & 2.2 & & 5.7 & & 0.0 & & \\
\hline Bifid uvula & & 2.2 & & 5.7 & & 0.0 & & \\
\hline Submucous cleft & & 3.3 & & 5.7 & & 1.8 & & \\
\hline High arched palate & 85 & 16.5 & 31 & 22.6 & 54 & 13.0 & 0.250 & a \\
\hline Ears, abnormal & 122 & 52.5 & 66 & 57.6 & 56 & 46.4 & 0.433 & a \\
\hline Low-set & & 9.8 & & 13.6 & & 5.4 & & \\
\hline Posterior rotated & & 7.4 & & 9.1 & & 5.4 & & \\
\hline Hypertrichosis & 128 & 86.7 & 76 & 94.7 & 52 & 75.0 & 0.001 & a \\
\hline \multicolumn{9}{|l|}{ Musculoskeletal anomalies } \\
\hline Scoliosis & 123 & 26.0 & 70 & 27.1 & 53 & 24.5 & 0.743 & a \\
\hline Pectus, excavatum & 104 & 13.5 & 57 & 14.0 & 47 & 12.8 & 0.850 & a \\
\hline Primary dentition, delayed & 65 & 44.6 & 40 & 50.0 & 25 & 36.0 & 0.313 & a \\
\hline Permanent dentition, delayed & 33 & 48.5 & 18 & 33.3 & 15 & 66.7 & 0.056 & a \\
\hline Widely spaced teeth & 72 & 41.7 & 40 & 40.0 & 32 & 43.8 & 0.748 & a \\
\hline Bone age, delayed & 40 & 47.5 & 30 & 46.7 & 10 & 50.0 & 1.000 & c \\
\hline Joint laxity & 88 & 60.2 & 52 & 61.5 & 36 & 58.3 & 0.763 & a \\
\hline Early arthritis & 75 & 5.3 & 36 & 5.6 & 39 & 5.1 & 1.000 & c \\
\hline Clinodactyly & 77 & 36.4 & 42 & 45.2 & 35 & 25.7 & 0.076 & a \\
\hline Short phalanges & 49 & 34.7 & 34 & 41.2 & 15 & 20.0 & 0.151 & a \\
\hline Complete absent or small 5th distal phalanx & 110 & 40.0 & 66 & 60.6 & 44 & 9.1 & 0.000 & c \\
\hline Prominent distal phalanges; & 102 & 24.5 & 64 & 31.3 & 38 & 13.2 & 0.040 & a \\
\hline Prominent interphalangeal joints & 103 & 21.4 & 64 & 28.1 & 39 & 10.3 & 0.032 & a \\
\hline Brachydactyly general & 60 & 16.7 & 19 & 15.8 & 41 & 17.1 & 1.000 & c \\
\hline Brachydactyly fifth finger & 68 & 30.9 & 22 & 50.0 & 46 & 21.7 & 0.018 & a \\
\hline Small nails & 122 & 54.9 & 73 & 68.5 & 49 & 34.7 & 0.000 & a \\
\hline Which nails, 5th finger and/or toe & 106 & 55.7 & 67 & 65.7 & 39 & 38.5 & 0.007 & a \\
\hline Which nails, all & 53 & 11.3 & 29 & 20.7 & 24 & 0.0 & 0.027 & c \\
\hline Fetal finger pads & 100 & 29.0 & 50 & 26.0 & 50 & 32.0 & 0.509 & a \\
\hline \multicolumn{9}{|l|}{ Intestinal } \\
\hline Inguinal hernia & 90 & 7.8 & 46 & 2.2 & 44 & 13.6 & 0.056 & c \\
\hline Intestinal problems & 105 & 48.6 & 60 & 36.7 & 45 & 64.4 & 0.000 & a \\
\hline Constipation & & 30.5 & & 21.7 & & 42.2 & & \\
\hline Gastroesophageal reflux & & 17.1 & & 13.3 & & 22.2 & & \\
\hline Diarrhea & & 4.8 & & 3.3 & & 6.7 & & \\
\hline Pyloric Stenosis & & 2.9 & & 5.0 & & 0.0 & & \\
\hline Umbilical hernia & & 4.8 & & 1.7 & & 8.9 & & \\
\hline
\end{tabular}


Table 1 continued

\begin{tabular}{|c|c|c|c|c|c|c|c|c|}
\hline \multirow[b]{2}{*}{ Clinical features $^{d}$} & \multirow{2}{*}{$\begin{array}{l}\text { Total } \\
n=143\end{array}$} & \multirow[b]{2}{*}{$\%$} & \multicolumn{2}{|c|}{ ARID1B-CSS } & \multicolumn{2}{|c|}{ ARID1B-ID } & \multirow[b]{2}{*}{$p$ value } & \multirow[b]{2}{*}{ Test } \\
\hline & & & $n=79$ & $\%$ & $n=64$ & $\%$ & & \\
\hline \multicolumn{9}{|l|}{ Cardiac \& genitourinary anomalies } \\
\hline Cardiac anomalies & 113 & 19.5 & 69 & 21.7 & 44 & 15.9 & 0.492 & a \\
\hline ASD & & 10.6 & & 13.0 & & 6.8 & & \\
\hline VSD & & 5.3 & & 5.8 & & 4.5 & & \\
\hline Renal anomalies & 95 & 12.6 & 53 & 11.3 & 42 & 14.3 & 0.666 & c \\
\hline Renal sonography, abnormal & 43 & 25.6 & 19 & 21.1 & 24 & 29.2 & 0.105 & c \\
\hline Cryptorchidism & 65 & 55.4 & 28 & 39.3 & 37 & 67.6 & 0.023 & a \\
\hline \multicolumn{9}{|l|}{ Endocrinological abnormalities ${ }^{e}$} \\
\hline Diabetes mellitus & 71 & 7.0 & 43 & 7.0 & 28 & 7.1 & 1.000 & c \\
\hline Type 2 diabetes mellitus & 4 & 75.0 & 2 & 100.0 & 2 & 50.0 & 1.000 & c \\
\hline Age (years) diagnosis (min-max) & 2 & $18-46$ & 1 & 18.0 & 1 & 46.0 & 0.317 & $\mathrm{~b}$ \\
\hline Hypothyroidism & 63 & 19.0 & 38 & 15.8 & 25 & 24.0 & 0.417 & a \\
\hline Age (years) diagnosis (median; min-max) & 10 & $8 ; 1-40$ & 4 & $4 ; 1.3-36.0$ & 6 & $6 ; 1.0-40.0$ & 0.394 & $\mathrm{~b}$ \\
\hline Growth hormone deficiency & 51 & 13.7 & 33 & 18.2 & 18 & 5.6 & 0.398 & c \\
\hline Growth hormone supplementation & 50 & 12.0 & 31 & 16.1 & 19 & 5.3 & 0.387 & c \\
\hline \multicolumn{9}{|l|}{ Behavioral characteristics } \\
\hline Behavioral abnormalities & 71 & 83.1 & 28 & 85.7 & 43 & 81.4 & 0.945 & a \\
\hline Hyperkinetic & & 15.5 & & 14.3 & & 16.3 & & \\
\hline Short attention & & 25.4 & & 25.0 & & 25.6 & & \\
\hline Impulsiveness & & 14.1 & & 14.3 & & 14.0 & & \\
\hline Obsessive & & 15.5 & & 14.3 & & 16.3 & & \\
\hline Rigid & & 8.5 & & 3.6 & & 11.6 & & \\
\hline Anger outbursts & & 16.9 & & 10.7 & & 20.9 & & \\
\hline Aggressive & & 16.9 & & 14.3 & & 18.6 & & \\
\hline Anxious & & 23.9 & & 17.9 & & 27.9 & & \\
\hline Poor sociability & & 19.7 & & 17.9 & & 20.9 & & \\
\hline Hyperactivity & 63 & 42.9 & 25 & 60.0 & 38 & 31.6 & 0.026 & a \\
\hline High pain threshold ${ }^{e}$ & 47 & 40.4 & 28 & 28.6 & 19 & 57.9 & 0.044 & a \\
\hline \multicolumn{9}{|l|}{ Psychiatric disorders } \\
\hline ADHD & 48 & 33.3 & 16 & 50.0 & 32 & 25.0 & 0.083 & a \\
\hline Autistic traits & 77 & 57.1 & 27 & 66.7 & 50 & 52.0 & 0.215 & a \\
\hline Malignancies & 97 & 1.0 & 53 & 0.0 & 44 & 2.3 & 0.454 & c \\
\hline
\end{tabular}

Only characteristics present in $\geq 5 \%$ of all patients or in either patient group, characteristics differing between groups, and distinctive features are shown.

$A D H D$ attention deficit hyperactivity disorder, ARID1B-CSS patient group with a suspicion of Coffin-Siris syndrome before genetic testing, ARID1B-ID patient group with no suspicion of Coffin-Siris syndrome before genetic testing, ASD atrial septal defect, CSS Coffin-Siris syndrome, EEG electroencephalography, ENT ear nose throat, OFC occipitofrontal circumference, SDS standard deviation score, VSD ventricular septal defect.

${ }^{a}$ Chi-square.

${ }^{\mathrm{b}}$ Mann-Whitney U.

'Fisher's.

dThe total number of a feature can differ from the sum of subcategories, because in some cases it was possible to answer with more than one option or to report the existence of a feature without specifying.

${ }^{\mathrm{e}}$ Data regarding these features were collected through email after first analyses.

are not statistically significant under the Bonferroni adjusted significance level.

Regarding all other features, no significant differences were found between the ARID1B-CSS and ARID1B-ID groups (Table 1). Therefore, in the remainder of this section no distinction is made between both groups.

\section{Overall phenotype}

\section{Growth and development}

Histograms of the standard deviations of height, weight, and OFC are shown in Fig. 2a-c. A height below -2 SDS was observed in $30.3 \%$. Confirming our previous data, ${ }^{11}$ the head circumference is normally distributed around 0 SDS and only $2.9 \%$ have an OFC below -2 SDS. Developmental milestones are shown in Fig. 2d, e. Speech, and gross and fine motor skills were delayed in almost all patients. Figure 2e shows the Kaplan-Meier plot of the age at first words, and this plot suggests that about $25 \%$ of patients do not develop speech.

Feeding difficulties were frequent $(69.4 \%)$ and led to tube feeding in $16.9 \%$ of patients. Bone age was delayed in $47.5 \%$ and scoliosis occurred in $26.0 \%$ of patients. Frequent 


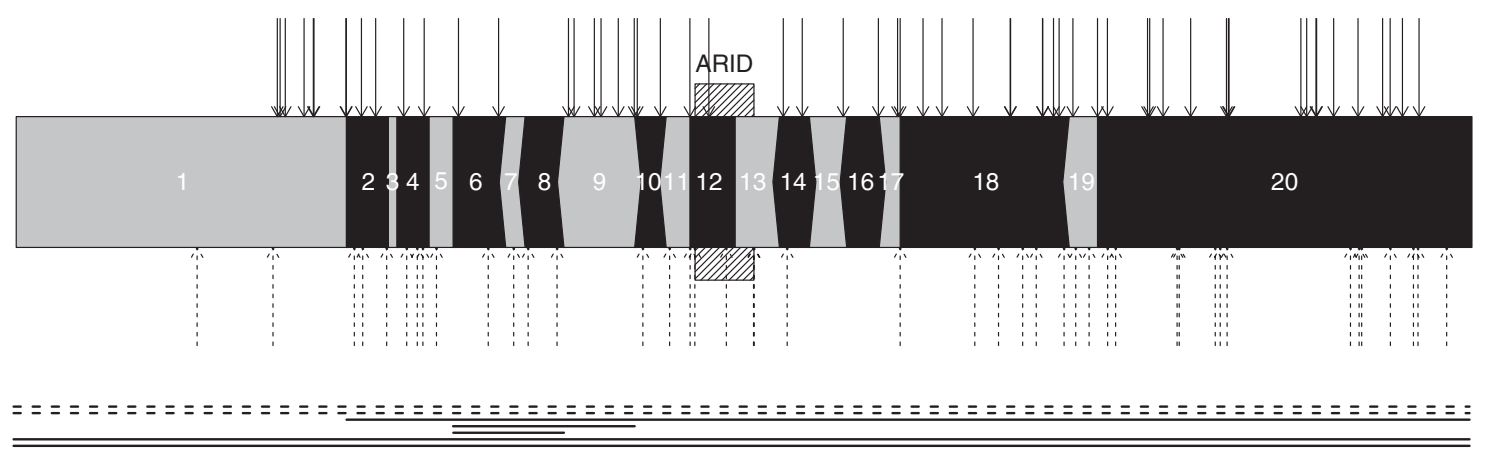

=-5-

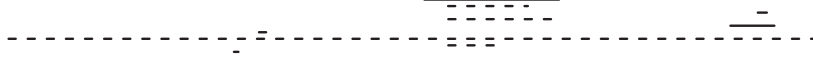

Fig. 1 Overview of the location of pathogenic variants in ARID1B. Numbers represent exon numbers and a graphical representation of in-frame and out-frame exons. When an exon ends with a complete codon, a vertical line is displayed. If it has one additional base an arrow to the left is displayed, and two additional bases are indicated by an arrow to the right. In-frame exons thus have the same boundary on both sides of the exon. Small variants (defined as events $\leq 20$ bases) are identified by the arrows above the exon structure; larger variants are shown as lines under the intron-exon structure. All large events were deletions. Only unique variants are shown. Uninterrupted lines represent variants in ARID1B-CSS patients; interrupted lines represent variants in ARID1B-ID patients.
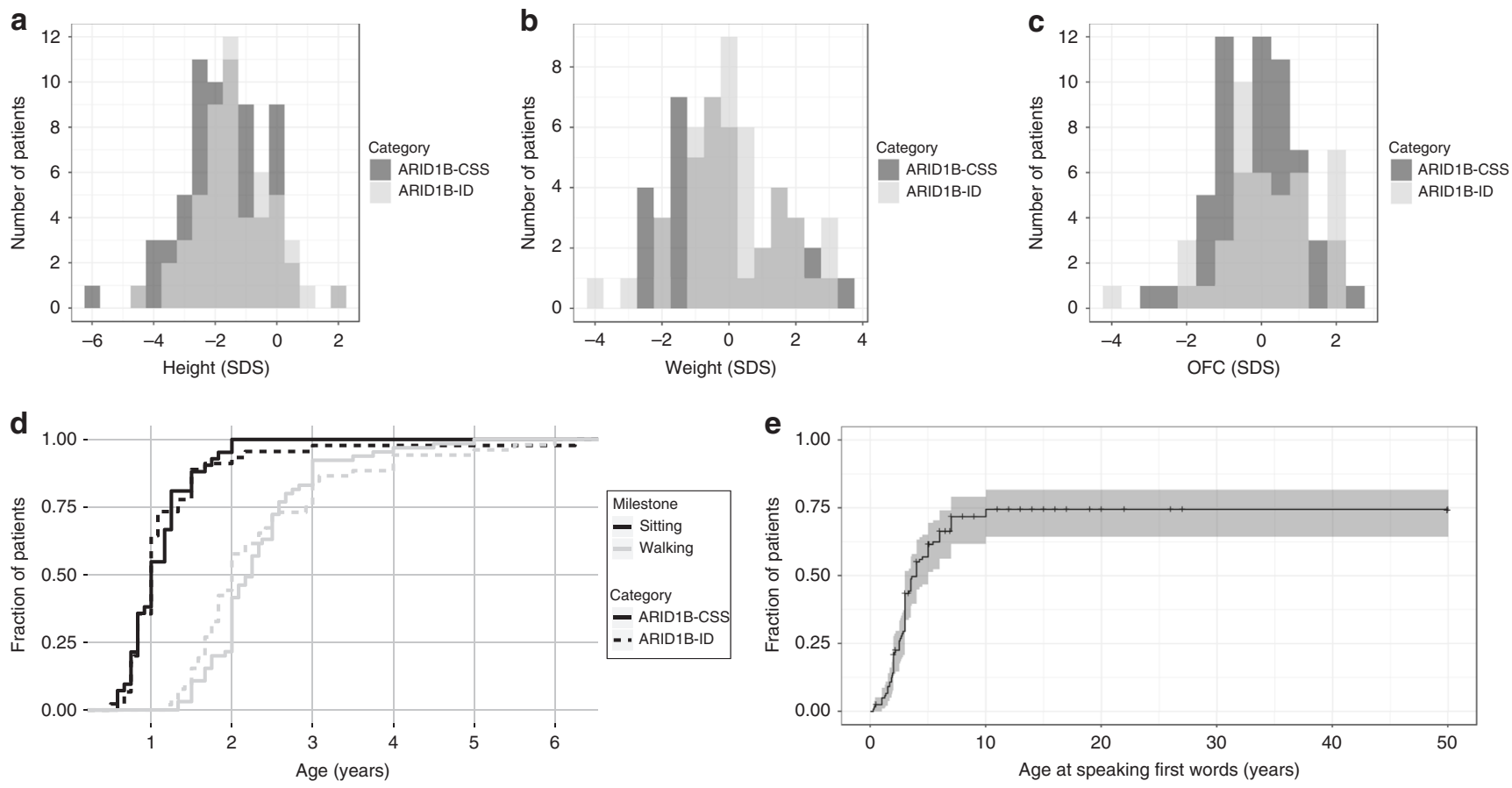

Fig. 2 Biometry and developmental milestones. Histograms of the standard deviation score (SDS) of (a) height, $n=122$; (b) weight, $n=92$; and (c) occipitofrontal circumference (OFC), $n=105$. (d) Cumulative distribution of the developmental milestones walking $(n=117)$ and sitting $(n=85)$ for ARID1B-CSS and ARID1B-ID. (e) Kaplan-Meier plot for the whole cohort of the age at which patients spoke their first words $(n=126)$. Confidence intervals of Kaplan-Meier plots are generated by R's survfit function.

infections (57.3\%) and gastrointestinal problems $(48.6 \%$, mostly constipation) were also reported.

\section{Congenital anomalies}

Cardiac anomalies (19.5\%), cryptorchidism (55.4\%), laryngomalacia (19.8\%), and a nonfunctioning or absent tear duct (15.1\%) were frequently reported. The cardiac anomalies consisted mostly of abnormalities/defects of the cardiac septa $(n=19)$ and/or mitral or aortic valves $(n=6)$. Renal anomalies were present in $12.6 \%$ of patients. The most frequent renal anomalies were hydronephrotic kidney $(n=3)$ and nephrolithiasis $(n=3)$.

\section{Neurological features}

Almost all patients exhibited a variable degree of ID. Figure 3a, b show the distribution of reported ID severity and IQ scores $(n=35)$. Remarkably, several patients did have an IQ in the normal range.

Hypotonia occurred in $81.0 \%$ of patients and $27.5 \%$ suffered from seizures, while an additional $5.6 \%$ had an abnormal 

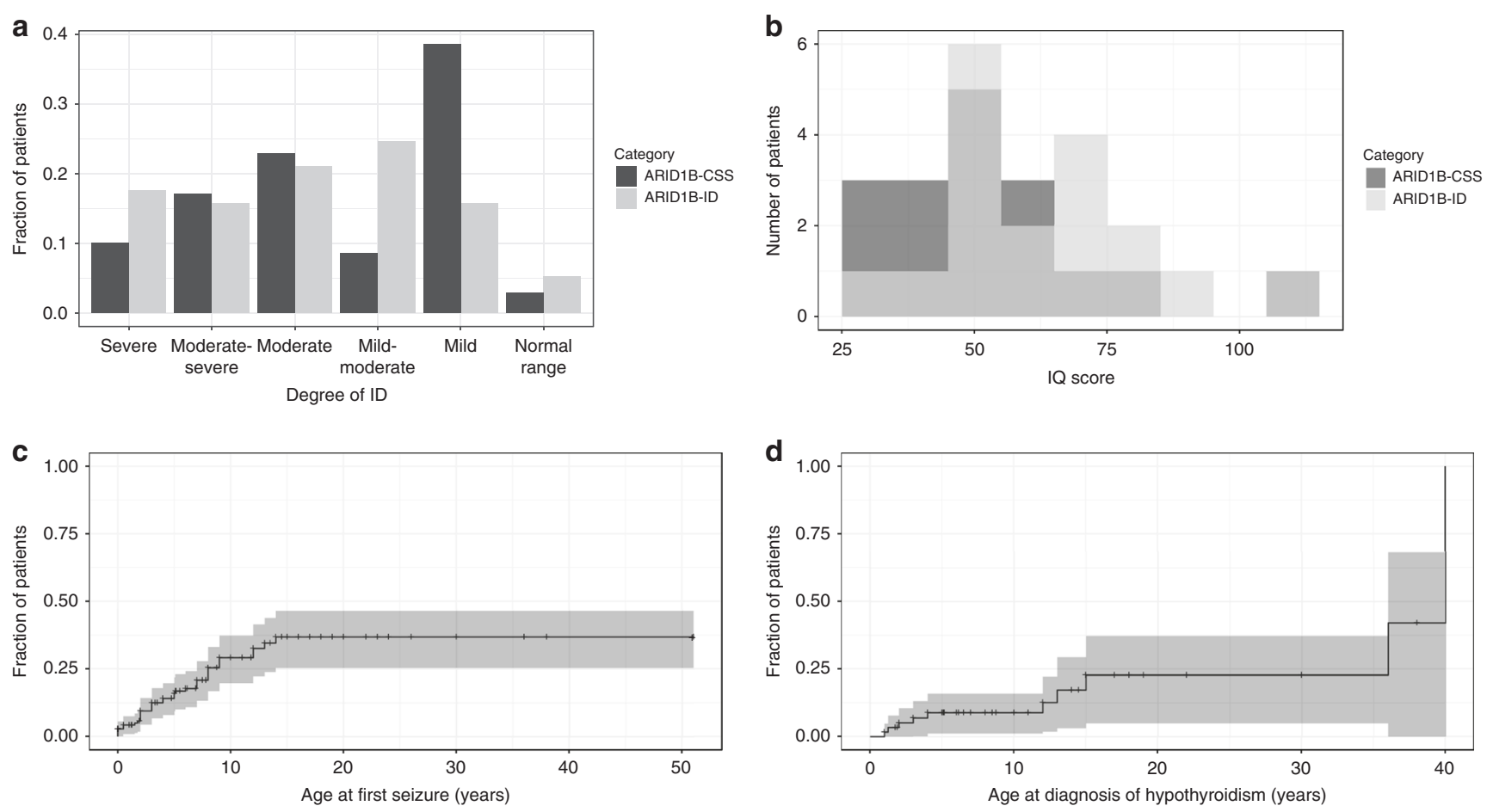

Fig. 3 Degree of intellectual disability and survival analyses of seizures and hypothyroidism. (a) Intellectual disability (ID) category as assessed by the treating physician, $n=127$; and (b) IQ scores (determined at different ages using different scales), $n=35$ for ARID1B-CSS and ARID1B-ID patients. (c) Kaplan-Meier plot for age of onset of seizures, $n=37$ and (d) Kaplan-Meier plot for the age at which hypothyroidism was diagnosed, $n=10$. Confidence intervals of Kaplan-Meier plots are generated by R's survfit function.

electroencephalogram (EEG). The age of onset of the seizures varied from 0 to 14 years, with a median of 4 years (Fig. $3 \mathrm{c}$ ). The Kaplan-Meier plot suggests that about $40 \%$ of patients are expected to experience one or more epileptic seizure during their life. All patients responded well to seizure medication $(n=18)$, while 4 did not receive medication. Complete or partial ACC (42.6\%) and delayed myelination (17.0\%) were the most prevalent cerebral anomalies.

\section{Visual and hearing impairment}

Vision problems (48.6\%) were twice as frequent as hearing problems (22.1\%). Myopia was the most reported vision problem (27.5\%); severity ranged from -24 to -1.0 (median -6.35). Hearing loss was congenital in most patients (63.6\%) and when present usually bilateral (51.9\%); four patients needed hearing aids.

\section{Endocrinological features}

Hypothyroidism was documented in $19.0 \%$ and one patient had hypothyroidism for 2 years, which spontaneously resolved. The Kaplan-Meier plot of age at which hypothyroidism developed (Fig. 3d) suggests that the prevalence may be $25 \%$, but because the numbers are small there is a large confidence interval. Of 71 individuals, 5 (7.0\%) were diagnosed with diabetes, 1 with hyperinsulinism, and 7/51 (13.7\%) had a growth hormone (GH) deficiency, of which 6 received GH supplementation. We had no information on whether GH deficiency was partial or complete.

\section{Effect of location of pathogenic variant}

The location of the pathogenic variant did not appear to correlate with the severity of the phenotype (Supplementary Figure S2). Because it has been reported that patients with pathogenic variants in in-frame exons sometimes have less severe phenotypes because of naturally occurring exon skipping, ${ }^{27}$ we compared the phenotypes of patients with pathogenic variants in in-frame exons versus those with pathogenic variants in out-frame exons. We classified patients with pathogenic variants in the first and last exon and wholegene deletions as out-frame, because such pathogenic variants cannot be rescued by exon skipping. Most pathogenic variants were classified as out-frame (84.6\%), which was expected because $86.1 \%$ of the coding region consists of out-frame exons. We did not find any differences in the phenotype between these two groups.

\section{Genotype}

\section{DISCUSSION}

Information on 143 patients with pathogenic variants in $A R I D 1 B$ was collected. In accordance with previous studies, all pathogenic variants were truncating (nonsense, frameshift, splice-site, and deletions of various numbers of exons including whole-gene deletions). In addition, four translocations $^{14,15,28,29}$ and three duplications ${ }^{2,16,21}$ affecting ARID1B have been reported in literature. While previous reviews ${ }^{30,31}$ have suggested that a pathogenic missense variant was reported in Tsurusaki et al., ${ }^{9}$ it is worth noting that a 
nonsense variant in $A R I D 1 B$ was also found in the same patient. Although parental DNA was not available for this patient, it seems most likely that the missense variant was harmless, while the nonsense variant was causal. Yu et al. ${ }^{16}$ described two pathogenic de novo missense variants in patients with short stature, but without ID or speech delay. Mignot et al. ${ }^{32}$ described a patient with mild ID and ACC who inherited a pathogenic missense variant from a mildly affected mother. Because the pathogenic variant (c.6092 $\mathrm{T}>\mathrm{C}$; p.Ile2031Thr) arose de novo in the mother, it is highly likely to be pathogenic. Thus, missense variants appear to be a much less common cause of ID than truncating variants. Missense variants identified in the absence of parental DNA should be interpreted with caution and are much more likely to be harmless. Care should also be taken with the interpretation of de novo missense variants in patients with ID.

As suggested by Johnston et al., ${ }^{33}$ it is important to take note if pathogenic variants in the same exon have been previously described when interpreting variants, because some annotated exons might in fact not be relevant for disease-associated transcripts. It is therefore noteworthy that we have not identified any pathogenic variants that only affect exon 3 (Fig. 1). Exon 3 is a small in-frame exon and we previously indicated ${ }^{11}$ that truncating variants in this exon are most likely benign. Indeed, Johnston et $\mathrm{al}^{33}$ report an inherited truncating variant in exon 3 in a patient without an ARID1B-related phenotype. Taking all evidence together, it is most likely that pathogenic variants in exon 3 are nonpathogenic, and that transcript NM_017519.2, which lacks exon 3 of NM_020732.3, is in fact the more relevant transcript. Another remarkable aspect that is apparent from Fig. 1 is that no pathogenic variants have been reported in the first 849 bases of $A R I D 1 B$. Although some frameshift variants are reported in gnomAD, these are dubious calls because the variants are in a low-complexity region. The lack of pathogenic variants may be by chance or due to sequencing difficulties in this GC-rich area, but it is also possible that there is an alternative start site that renders truncating variants in the first part of exon 1 neutral. Therefore, we advise caution when interpreting such variants.

Although a previous analysis seemed to suggest that pathogenic variants in the last exon might result in a more severe phenotype, ${ }^{11}$ repeating this analysis with the current data did not confirm this suspicion (Supplementary Figure S2).

\section{Penetrance}

Penetrance of rare variants cannot be directly estimated, but the inheritance status and prevalence of apparently pathogenic variants in population databases can be used as proxy. ${ }^{34}$ Pathogenic variants were de novo in all cases where inheritance could be established $(n=107)$. We did observe two sisters with the same pathogenic variant, and although this could be due to paternal inheritance, the most likely explanation is gonadal mosaicism, as previously described for ARID1B. ${ }^{17}$
The gnomAD browser may be viewed as a large population data set, consisting of exomes and genomes of unrelated, unaffected individuals sequenced as part of disease-specific and population genetic studies. In transcript NM_020732.2 (ENST00000346085), fewer missense variants in ARID1B are reported than would be expected if these were to occur randomly (ExAC accessed 10 April 2018; 744.4 expected, 555 observed, $z$-score of 3.39). Ten loss-of-function variants are reported; half of these might not be pathogenic because they are either in the start of exon 1, or in exon 3 (Supplementary Table S5). The other five variants, three splice-site variants and two frameshift variants, are potentially pathogenic. This might suggest that the penetrance of pathogenic variants in $A R I D 1 B$ is not complete, and that these individuals had an average IQ as some of the individuals in our study. Therefore, while the penetrance of pathogenic variants in $A R I D 1 B$ appears to be very high, we still recommend parental testing, especially when future pregnancies are considered.

\section{Phenotype}

The main differences between ARID1B-CSS and ARID1B-ID seem to be related to dysmorphic features. Therefore, we conclude from this data set that the ARID1B-related disorders represent a spectrum. Not every feature is present in all patients, and depending on the combination of features present, and the experience of the clinician concerned, a patient might receive a clinical diagnosis of CSS. Although the patients in our previous $A R I D 1 B-C S S$ cohort $^{11}$ had an equal sex ratio, our current cohort finds significantly more females than males in the ARID1B-CSS group compared with the ARID1B-ID group. This may be because some features, most notably hypertrichosis, are easier to recognize as abnormal in females than in males.

\section{Endocrinological features}

Hypothyroidism was reported in $12 / 63$ patients, and most were diagnosed before the age of 15 years (Fig. 3d). Diabetes was diagnosed in 5/71 patients; 4 of these patients were reported to have type 2 diabetes and had a relatively high weight, while one was diagnosed with type 1 diabetes, and 1 patient was diagnosed with hyperinsulinism. This patient with hyperinsulinism has been described previously, ${ }^{19}$ and one additional patient has been reported to have hyperinsulinism. $^{10}$ Of our patients, 7/51 were diagnosed with $\mathrm{GH}$ deficiency, and 6 of those received GH supplementation. Likewise, several patients with a growth delay due to $\mathrm{GH}$ deficiency were described ${ }^{16}$ and a similar phenomenon was replicated in an Arid1b heterozygous mouse model. ${ }^{35}$ Considering $30 \%$ of patients had short stature $\left(<-2\right.$ SDS), ${ }^{36}$ GH deficiency could be an underrecognized feature of ARID1B patients.

\section{Cancer}

Somatic variants in $A R I D 1 B$ have been associated with several types of cancer. ${ }^{37}$ However, only one case is known of a patient with a pathogenic germline variant in $A R I D 1 B$ and (thyroid) cancer. $^{38}$ Similarly, in our cohort only one patient had 
malignancies. This boy had a Sertoli-Leydig cell tumor at the age of 3 and a temporal glioneuronal tumor at 12 years of age. No genetic testing was performed in this patient to detect the presence of specific tumor syndromes. Based on our patient cohort it seems unlikely that pathogenic germline variants in $A R I D 1 B$ confer an increased cancer risk, but longer follow-up of our patients is needed to make a definitive statement.

\section{Phenotype delineation methods and biases}

The increased awareness of ascertainment bias and the increasing number of newly discovered disease genes have resulted in new methods for data collection. Paper questionnaires and Excel spreadsheets are rapidly being replaced by Internet forms and HPO-based methods along with more formal registry software interfaces such as PhenoTips. ${ }^{39}$ For the current study we have chosen to use an online questionnaire based on our previous data, with mostly fixed answers. More general genetic databases (such as DECIPHER ${ }^{40}$ ) accommodate inclusion of phenotypic data by using HPO terms. Whereas HPO-based methods allow for more straightforward identification of new, unexpected findings, our approach with specific questions allows calculation of true frequencies of features, because we can discriminate between absence of a feature and missing data. To investigate whether this influences results, we compared our data regarding the phenotype of ARID1B patients with the features reported for ARID1B patients in the DECIPHER database. It should be noted that there are some patients who are both in our cohort and the DECIPHER database. On 2 October 2017 DECIPHER contained 54 openaccess ARID1B patients with 247 phenotypic features. Except for laryngomalacia and excessive salivation and/or drooling, all characteristics enriched in ARID1B patients in DECIPHER had been included in our questionnaire. The DECIPHER frequency of all reported features was lower than ours, a difference that was statistically significant for most (Supplementary Table S6).

In addition to other phenotypic features, growth and development are graphically represented on the DECIPHER website. We replicated some of these graphs and found only minor differences, which may be explained by the increased volume of data available to us (Supplementary Table S7 and Supplementary Figure S3).

Based on these results, we conclude that the DECIPHER database is a valuable tool to assess the potential features that ought to be included in gene-specific phenotype questionnaires, but the reported percentages are potential underestimations, likely due to phenotype underreporting bias owing to their data collection method. This bias could be mitigated by developing an adaptive questionnaire, so that submitters are requested to include or actively exclude features that have been mentioned several times in patients with the same underlying genetic cause.

Another bias that is present in most cohorts is an agerelated bias. Most of our population is young, and this precludes us from detecting features that typically present at later age. This bias can partially be resolved by performing survival analysis, as we have done for speech, seizures, and hypothyroidism. In all cases there was a clear difference in our global frequency estimate, and the estimate provided by the Kaplan-Meier plot. We therefore recommend that authors of clinical cohorts include the age of occurrence of agedependent features and perform survival analysis.

\section{Study limitations}

Our study has several limitations. Data entry was performed by many different clinicians, which could have led to different interpretation of subjective questions, such as whether the patient fits the CSS spectrum. During this study, it became evident from literature and the DECIPHER database that previously unknown features, such as laryngomalacia, were part of the ARID1B spectrum and should have been added to our questionnaire. The effect of these incompletions was mitigated in part by the presence of an open field and our request to the contributing clinicians to send us an update on their patient's characteristics for frequently reported additional features.

Although we have attempted to account for different sources of bias, we cannot exclude that we currently overestimate the presence of some features, because our calculation is based on those patients having the feature, divided by those who are reported not to have the feature. It is possible that in some cases the absence of a feature is not consciously recognized or recorded in the clinical charts, which may result in scoring "unknown" rather than "absent" for a given feature. This bias can only be mitigated by thoroughly phenotyping every patient by a limited number of physicians, and is something we are planning to do with our national CSS cohort in the near future.

\section{Conclusion}

We conclude that the ARID1B-related disorders encompass a spectrum of features. The typical ARID1B patient has ID, feeding difficulties, laryngomalacia, speech delay, motor delay, hypertrichosis, and cryptorchidism. Our data suggest that endocrinological abnormalities, in particular hypothyroidism, may also be part of the ARID1B spectrum, but further research is needed to confirm this finding. There are few differences between ARID1B-CSS and ARID1B-ID, and we recommend that patients should be managed similarly. Based on the clinical data presented here we update our previous recommendations ${ }^{11}$ for the management of ARID1B patients:

\section{At the time of diagnosis}

- Rule out congenital anomalies by performing renal and cardiac ultrasounds. Thoroughly check for cryptorchidism and laryngomalacia as indicated.

- Refer to clinical geneticist for counseling.

\section{At the time of diagnosis and at follow-up}

- Evaluate growth; consider referral to endocrinologist when significantly delayed.

- Evaluate hearing and vision regularly. 
- Consider an EEG if there is a suspicion of seizures.

- Evaluate feeding problems. Offer dietary advice and feeding management. Consider evaluation by a gastroenterologist and/or a swallowing study.

- Treat constipation adequately.

- If indicated, early intervention using speech and/or physical therapy.

- Yearly evaluation of development by a specialized pediatrician and implementation of a specialized education plan for school or daycare as indicated.

- Periodic evaluations for scoliosis.

- On indication, determine thyroid status and glucose concentrations.

We also recommend periodic evaluation by a team of professionals specializing in ARID1B-related disorders (e.g., pediatrician, pediatric physiatrist, physical therapist, speech therapist, behavioral specialist, pediatric neurologist, clinical geneticist), which may take place at a distance.

\section{ELECTRONIC SUPPLEMENTARY MATERIAL}

The online version of this article (https://doi.org/10.1038/s41436$018-0330-z)$ contains supplementary material, which is available to authorized users.

\section{ACKNOWLEDGEMENTS}

We are grateful for the assistance of Pepijn Cox in setting up the website www.arid1bgene.com. This study has made use of data generated by the Human Disease Genes website series, www. humandiseasegenes.com. This work was financially supported by grants from the Netherlands Organisation for Health Research and Development (917-86-319 to B.B.A.d.V., 912-12-109 to B.B.A.d.V.)

\section{DISCLOSURE}

The authors declare no conflicts of interest.

\section{REFERENCES}

1. Wright CF, Fitzgerald TW, Jones WD, et al. Genetic diagnosis of developmental disorders in the DDD study: a scalable analysis of genomewide research data. Lancet. 2015;385:1305-14.

2. Hoyer J, Ekici AB, Endele S, et al. Haploinsufficiency of ARID1B, a member of the SWI/SNF-a chromatin-remodeling complex, is a frequent cause of intellectual disability. Am J Hum Genet. 2012;90:565-72.

3. Deciphering Developmental Disorders Study. Large-scale discovery of novel genetic causes of developmental disorders. Nature . 2015;519:223-8.

4. Santen GW, Aten E, Vulto-van Silfhout AT, et al. Coffin-Siris syndrome and the BAF complex: genotype-phenotype study in 63 patients. Hum Mutat. 2013:34:1519-28.

5. Wieczorek D, Bogershausen N, Beleggia F, et al. A comprehensive molecular study on Coffin-Siris and Nicolaides-Baraitser syndromes identifies a broad molecular and clinical spectrum converging on altered chromatin remodeling. Hum Mol Genet. 2013;22:5121-35.

6. Tsurusaki Y, Okamoto N, Ohashi $\mathrm{H}$, et al. Coffin-Siris syndrome is a SWI/ SNF complex disorder. Clin Genet. 2014;85:548-54.

7. Schrier Vergano S, Santen G, Wieczorek D, Wollnik B, Matsumoto N, Deardorff MA. Coffin-Siris syndrome. In: Pagon RA, Adam MP, Ardinger $\mathrm{HH}$, et al., eds. GeneReviews. Seattle (WA) University of Washington 1993.

8. Santen GW, Aten E, Sun Y, et al. Mutations in SWI/SNF chromatin remodeling complex gene ARID1B cause Coffin-Siris syndrome. Nat Genet. 2012;44:379-80.
9. Tsurusaki $\mathrm{Y}$, Okamoto $\mathrm{N}$, Ohashi $\mathrm{H}$, et al. Mutations affecting components of the SWI/SNF complex cause Coffin-Siris syndrome. Nat Genet. 2012;44:376-8.

10. Vals MA, Oiglane-Shlik E, Noukas $M$, et al. Coffin-Siris syndrome with obesity, macrocephaly, hepatomegaly and hyperinsulinism caused by a mutation in the ARID1B gene. Eur J Hum Genet. 2014;22:1327-9.

11. Santen GW, Clayton-Smith J. ARID1B-CSS consortium. The ARID1B phenotype: what we have learned so far. Am J Med Genet C Semin Med Genet. 2014;166C:276-89.

12. Mari F, Marozza A, Mencarelli MA, et al. Coffin-Siris and NicolaidesBaraitser syndromes are a common well recognizable cause of intellectual disability. Brain Dev. 2015;37:527-36.

13. Nord AS, Roeb W, Dickel DE, et al. Reduced transcript expression of genes affected by inherited and de novo CNVs in autism. Eur J Hum Genet. 2011;19:727-31.

14. Halgren C, Kjaergaard S, Bak M, et al. Corpus callosum abnormalities, intellectual disability, speech impairment, and autism in patients with haploinsufficiency of ARID1B. Clin Genet. 2012;82:248-55.

15. Sim JC, White SM, Fitzpatrick $E$, et al. Expanding the phenotypic spectrum of ARID1B-mediated disorders and identification of altered cellcycle dynamics due to ARID1B haploinsufficiency. Orphanet J Rare Dis. 2014:9:43.

16. $Y u Y, Y a o R$, Wang $L$, et al. De novo mutations in ARID1B associated with both syndromic and nonsyndromic short stature. BMC Genom. 2015; 16:701.

17. Ben-Salem S, Sobreira N, Akawi NA, et al. Gonadal mosaicism in ARID1B gene causes intellectual disability and dysmorphic features in three siblings. Am J Med Genet A. 2016;170A:156-61.

18. Smith JA, Holden KR, Friez MJ, Jones JR, Lyons MJ. A novel familial autosomal dominant mutation in ARID1B causing neurodevelopmental delays, short stature, and dysmorphic features. Am J Med Genet A. 2016;170:3313-8.

19. Sonmez FM, Uctepe E, Gunduz M, et al. Coffin-Siris syndrome with cafeau-lait spots, obesity and hyperinsulinism caused by a mutation in the ARID1B gene. Intractable Rare Dis Res. 2016;5:222-6.

20. Takenouchi T, Yoshihashi H, Sakaguchi $Y$, et al. Hirschsprung disease as a yet undescribed phenotype in a patient with ARID1B mutation. Am J Med Genet A. 2016;170:3249-52.

21. Seabra CM, Szoko N, Erdin S, et al. A novel microduplication of ARID1B: clinical, genetic, and proteomic findings. Am J Med Genet A. 2017;173:2478-84

22. Zahir FR, Mwenifumbo JC, Chun HE, et al. Comprehensive whole genome sequence analyses yields novel genetic and structural insights for intellectual disability. BMC Genom. 2017;18:403.

23. Zweier M, Peippo MM, Poyhonen M, et al. The HHID syndrome of hypertrichosis, hyperkeratosis, abnormal corpus callosum, intellectual disability, and minor anomalies is caused by mutations in ARID1B. Am J Med Genet A. 2017;173:1440-3.

24. Trujillano D, Bertoli-Avella AM, Kumar Kandaswamy K, et al. Clinical exome sequencing: results from 2819 samples reflecting 1000 families. Eur J Hum Genet. 2017;25:176-82.

25. Fenton TR, Kim JH. A systematic review and meta-analysis to revise the Fenton growth chart for preterm infants. BMC Pediatr. 2013;13:59.

26. Baban A, Moresco L, Divizia MT, et al. Pituitary hypoplasia and growth hormone deficiency in Coffin-Siris syndrome. Am J Med Genet A. 2008;146A:384-8.

27. Kapahnke M, Banning A, Tikkanen R. Random splicing of several exons caused by a single base change in the target exon of CRISPR/Cas9 mediated gene knockout. Cells. 2016;5:E45.

28. Backx L, Seuntjens E, Devriendt K, Vermeesch J, Van Esch H. A balanced translocation $t(6 ; 14)$ (q25.3; q13.2) leading to reciprocal fusion transcripts in a patient with intellectual disability and agenesis of corpus callosum. Cytogenet Genome Res. 2011;132:135-43.

29. Malli T, Duba HC, Erdel M, et al. Disruption of the ARID1B and ADAMTS6 loci due to a $t(5 ; 6)(q 12.3 ; q 25.3)$ in a patient with developmental delay. Am J Med Genet A. 2014;164A:3126-31.

30. Sim JC, White SM, Lockhart PJ. ARID1B-mediated disorders: mutations and possible mechanisms. Intractable Rare Dis Res. 2015;4:17-23.

31. Son EY, Crabtree GR. The role of BAF (mSWI/SNF) complexes in mammalian neural development. Am J Med Genet C Semin Med Genet. 2014;166C:333-49.

32. Mignot C, Moutard ML, Rastetter A, et al. ARID1B mutations are the major genetic cause of corpus callosum anomalies in patients with intellectual disability. Brain. 2016:e64. 
33. Johnston JJ, Lewis $\mathrm{KL}, \mathrm{Ng} \mathrm{D}$, et al. Individualized iterative phenotyping for genome-wide analysis of loss-of-function mutations. Am J Hum Genet. 2015;96:913-25.

34. Rosenfeld JA, Coe BP, Eichler EE, Cuckle H, Shaffer LG. Estimates of penetrance for recurrent pathogenic copy-number variations. Genet Med. 2013;15:478-81.

35. Celen $C$, Chuang JC, Luo $X$, et al. Arid $1 b$ haploinsufficient mice reveal neuropsychiatric phenotypes and reversible causes of growth impairment. Elife. 2017;6:e25730.

36. Pedicelli S, Peschiaroli E, Violi E, Cianfarani S. Controversies in the definition and treatment of idiopathic short stature (ISS). J Clin Res Pediatr Endocrinol. 2009:1:105-15.

37. Biegel JA, Busse TM, Weissman BE. SWI/SNF chromatin remodeling complexes and cancer. Am J Med Genet C Semin Med Genet. 2014;166C:350-66.

38. Vengoechea J, Carpenter L, Zarate YA. Papillary thyroid cancer in a patient with interstitial $6 q 25$ deletion including ARID1B. Am J Med Genet A. 2014;164A:1857-9.

39. Girdea M, Dumitriu S, Fiume $M$, et al. PhenoTips: patient phenotyping software for clinical and research use. Hum Mutat. 2013;34:1057-65.
40. Firth HV Richards SM, Bevan AP, et al. DECIPHER: Database of Chromosomal Imbalance and Phenotype in Humans Using Ensembl Resources. Am J Hum Genet. 2009;84:524-33.

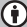

Open Access This article is licensed under a Creative Commons Attribution 4.0 International License, which permits use, sharing, adaptation, distribution and reproduction in any medium or format, as long as you give appropriate credit to the original author(s) and the source, provide a link to the Creative Commons license, and indicate if changes were made. The images or other third party material in this article are included in the article's Creative Commons license, unless indicated otherwise in a credit line to the material. If material is not included in the article's Creative Commons license and your intended use is not permitted by statutory regulation or exceeds the permitted use, you will need to obtain permission directly from the copyright holder. To view a copy of this license, visit http://creativecommons.org/licenses/ by/4.0\%

(c) The Author(s) 2018

Pleuntje J. van der Sluijs, MSc${ }^{1}$, Sandra Jansen, MD², Samantha A. Vergano, MD ${ }^{3}$, Miho Adachi-Fukuda, MD ${ }^{4}$, Yasemin Alanay, MD, PhD ${ }^{5}$, Adila AlKindy, MD ${ }^{6}$, Anwar Baban, MD, PhD ${ }^{7}$ Allan Bayat, MD, PhD ${ }^{8}$, Stefanie Beck-Wödl, PhD ${ }^{9}$, Katherine Berry, MS ${ }^{10}$, Emilia K. Bijlsma, MD, PhD ${ }^{1}$, Levinus A. Bok, MD, PhD ${ }^{11}$, Alwin F. J. Brouwer, $M D, P^{12}$, Ineke van der Burgt, $M D, P^{13}$ Philippe M. Campeau, MD ${ }^{14}$, Natalie Canham, MD ${ }^{15,16}$, Krystyna Chrzanowska, MD, PhD ${ }^{17}$, Yoyo W. Y. Chu, MS ${ }^{18}$, Brain H. Y. Chung, MD ${ }^{18}$, Karin Dahan, MD, PhD ${ }^{19}$, Marjan De Rademaeker, $\mathrm{MD}^{20}$, Anne Destree, $\mathrm{MD}^{19}$, Tracy Dudding-Byth, MD, PhD ${ }^{21}$, Rachel Earl, BSC ${ }^{22}$, Nursel Elcioglu, MD ${ }^{23}$, Ellen R. Elias, MD ${ }^{24}$, Christina Fagerberg, MD $^{25}$, Alice Gardham, MD ${ }^{15}$, Blanca Gener, $\mathrm{MD}^{26}$, Erica H. Gerkes, MD ${ }^{27}$, Ute Grasshoff, MD ${ }^{9}$, Arie van Haeringen, $\mathrm{MD}^{1}$, Karin R. Heitink, $\mathrm{BHS}^{28}$, Johanna C. Herkert, $\mathrm{MD}^{27}$,

Nicolette S. den Hollander, MD, PhD ${ }^{1}$, Denise Horn, $\mathrm{MD}^{29}$, David Hunt, MD, $\mathrm{PhD}^{30}$,

Sarina G. Kant, MD, PhD ${ }^{1}$, Mitsuhiro Kato, MD, PhD ${ }^{31}$, Hülya Kayserili, MD, PhD ${ }^{32}$,

Rogier Kersseboom, MD, PhD ${ }^{33}$, Esra Kilic, $\mathrm{MD}^{34}$, Malgorzata Krajewska-Walasek, MD, PhD ${ }^{17}$

Kylin Lammers, $\mathrm{MS}^{35}$, Lone W. Laulund, $\mathrm{MD}^{36}$, Damien Lederer, MD, PhD ${ }^{19}$, Melissa Lees, $\mathrm{MD}^{37}$,

Vanesa López-González, MD ${ }^{38}$, Saskia Maas, MD ${ }^{39}$, Grazia M. S. Mancini, MD, PhD ${ }^{33}$,

Carlo Marcelis, MD ${ }^{2}$, Francisco Martinez, $\mathrm{PhD}^{40}$, Isabelle Maystadt, MD, PhD ${ }^{19}$

Marianne McGuire, MD ${ }^{41}$, Shane McKee, MD ${ }^{42}$, Sarju Mehta, MD ${ }^{43}$, Kay Metcalfe, MD ${ }^{44}$,

Jeff Milunsky, MD ${ }^{45}$, Seiji Mizuno, MD ${ }^{46}$, John B. Moeschler, MD ${ }^{47}$, Christian Netzer, MD ${ }^{48}$,

Charlotte W. Ockeloen, MD, PhD², Barbara Oehl-Jaschkowitz, MD ${ }^{49}$, Nobuhiko Okamoto, MD, PhD ${ }^{50}$, Sharon N. M. Olminkhof, MSc ${ }^{51}$, Carmen Orellana, $\mathrm{PhD}^{40}$, Laurent Pasquier, MD ${ }^{52}$,

Caroline Pottinger, $\mathrm{MD}^{53}$, Vera Riehmer, $\mathrm{PhD}^{48}$, Stephen P. Robertson, Md, PhD ${ }^{54}$,

Maian Roifman, MD ${ }^{55,56}$, Caroline Rooryck, MD, PhD ${ }^{57}$, Fabienne G. Ropers, MD ${ }^{58}$,

Monica Rosello, MD, PhD ${ }^{40}$, Claudia A. L. Ruivenkamp, PhD ${ }^{1}$, Mahmut S. Sagiroglu, PhD ${ }^{59}$

Suzanne C. E. H. Sallevelt, MD, PhD ${ }^{60}$, Amparo Sanchis Calvo, MD ${ }^{61}$, Pelin O. Simsek-Kiper, MD, PhD ${ }^{62}$, Gabriela Soares, $\mathrm{MD}^{63}$, Lucia Solaeche, $\mathrm{MD}^{64}$, Fatma Mujgan Sonmez, MD ${ }^{65}$, Miranda Splitt, $\mathrm{MD}^{66}$, Duco Steenbeek, MD, $\mathrm{PhD}^{28}$, Alexander P. A. Stegmann, $\mathrm{PhD}^{60}$,

Constance T. R. M. Stumpel, MD, PhD ${ }^{60}$, Saori Tanabe, MD ${ }^{67}$, Eyyup Uctepe, MD ${ }^{68}$, G. Eda Utine, MD ${ }^{62}$, Hermine E. Veenstra-Knol, MD ${ }^{27}$, Sunita Venkateswaran, MD ${ }^{69}$, Catheline Vilain, MD ${ }^{70,71}$, Catherine Vincent-Delorme, MD ${ }^{72}$, Anneke T. Vulto-van Silfhout, MD, PhD ${ }^{2}$, Patricia Wheeler, MD ${ }^{73}$, Golder N. Wilson, MD, PhD ${ }^{74}$, Louise C. Wilson, MD ${ }^{37}$, Bernd Wollnik, MD ${ }^{75}$,

Tomoki Kosho, MD, PhD ${ }^{76}$, Dagmar Wieczorek, MD ${ }^{77}$, Evan Eichler, PhD ${ }^{78}$, Rolph Pfundt, $\mathrm{PhD}^{2}$,

Bert B. A. de Vries, MD, PhD ${ }^{2}$, Jill Clayton-Smith, MD ${ }^{44}$ and Gijs W. E. Santen, MD, PhD ${ }^{1}$ 
${ }^{1}$ Department of Clinical Genetics, Leiden University Medical Center, Leiden, The Netherlands. ${ }^{2}$ Department of Human Genetics, Donders Institute for Brain, Cognition and Behaviour, Radboud University Medical Center, Nijmegen, The Netherlands. ${ }^{3}$ Division of Medical Genetics and Metabolism, Children's Hospital of the King's Daughters, Norfolk, VA, USA. ${ }^{4}$ Department of Pediatrics, St. Marianna University School of Medicine, Kanagawa, Japan. ${ }^{5}$ School of Medicine, Department of Pediatrics, Pediatric Genetics Unit, Acibadem University, Istanbul, Turkey. ${ }^{6}$ Department of Genetics, Sultan Qaboos University Hospital, Muscat, Oman. ${ }^{7}$ Pediatric Cardiology and Cardiac Surgery Department, Bambino Gesù Children Hospital and Research Institute, IRCCS, Rome, Italy. ${ }^{8}$ Copenhagen University Hospital Hvidovre, Copenhagen, Denmark. ${ }^{9}$ Department of Molecular Genetics and Applied Genomics, University Hospital Tübingen, Tübingen, Germany. ${ }^{10}$ Department of Medical Genetics, Shodair Hospital, Helena, MT, USA. ${ }^{11}$ Department of Pediatrics, Màxima Medical Centre, Veldhoven, The Netherlands. ${ }^{12}$ Department of Paediatrics, Nij Smellinghe Hospital, Drachten, The Netherlands. ${ }^{13}$ Department of Human Genetics, Radboud University Medical Center, Nijmegen, The Netherlands. ${ }^{14}$ Department of Pediatrics, CHU Sainte-Justine and University of Montreal, Montreal, QC, Canada. ${ }^{15}$ North West Thames Regional Genetics Service, Northwick Park Hospital, Harrow, United Kingdom. ${ }^{16}$ Cheshire and Merseyside Regional Genetics Service, Liverpool Women's Hospital, Crown Street, Liverpool, United Kingdom. ${ }^{17}$ Department of Medical Genetics, The Children's Memorial Health Institute, Warsaw, Poland. ${ }^{18}$ Department of Paediatrics and Adolescent Medicine, Li Ka Shing Faculty of Medicine, The University of Hong Kong, Hong Kong, SAR, China. ${ }^{19}$ Center for Human Genetics, Institute of Pathology and Genetics, Gosselies, Belgium. ${ }^{20}$ Center for Medical Genetics, Vrije Universiteit Brussels, Brussels, Belgium. ${ }^{21}$ Hunter Genetics and University of Newcastle, GrowUpWell Priority Research Centre, Newcastle, Australia. ${ }^{22}$ Department of Psychiatry and Behavioral Sciences, University of Washington, Seattle, WA, USA. ${ }^{23}$ Department of Pediatric Genetics, Marmara University Pendik Hospital, Istanbul, Turkey. ${ }^{24}$ Department of Pediatrics and Genetics, University of Colorado Denver School of Medicine, Aurora, CO, USA.

${ }^{25}$ Department of Clinical Genetics, Odense University Hospital, Odense, Denmark. ${ }^{26}$ Department of Genetics, Cruces University Hospital, Biocruces Health Research Institute, Vizcayam, Spain. ${ }^{27}$ University of Groningen, University Medical Center Groningen, Department of Genetics, Groningen, The Netherlands. ${ }^{28}$ Department of Rehabilitation Medicine, Leiden University Medical Center, Leiden, The Netherlands. ${ }^{29}$ Institute for Medical Genetics and Human Genetics, Charité Universitätsmedizin, Berlin, Germany. ${ }^{30}$ Wessex Clinical Genetics Service, Princess Anne Hospital, Southampton, United Kingdom. ${ }^{31}$ Department of Pediatrics, Showa University School of Medicine, Tokyo, Japan. ${ }^{32}$ Medical Genetics Department, Koç University School of Medicine (KUSoM), İstanbul, Turkey. ${ }^{33}$ Department of Clinical Genetics, Sophia Children's Hospital, Erasmus MC, Rotterdam, The Netherlands. ${ }^{34}$ Department of Pediatric Genetics, Hematology Oncology Research \& Training Children's Hospital, Ankara, Turkey. ${ }^{35}$ Department of Medical Genetics, Dayton Children's Hospital, Dayton, OH, USA. ${ }^{36}$ Department of Paediatrics, Odense University Hospital, Odense, Denmark. ${ }^{37}$ Department of Clinical Genetics, Great Ormond Street Hospital NHS Foundation Trust, London, United Kingdom. ${ }^{38}$ Sección de Genética Médica, Servicio de Pediatria, Hospital Clinico Universitario Virgen de la Arrixaca, IMIB-Arrixaca, CIBERERISCIII, Murcia, Spain. ${ }^{39}$ Department of Clinical Genetics, Academic Medical Center, University of Amsterdam, Amsterdam, The Netherlands. ${ }^{40}$ Unidad de Genética, Hospital Universitario y Politécnico La Fe, Valencia, Spain. ${ }^{41}$ Department of Molecular and Human Genetics, Baylor College of Medicine, One Baylor Plaza, Houston, TX, USA. ${ }^{42}$ Northern Ireland Regional Genetics Centre, Belfast City Hospital, Belfast, Ireland. ${ }^{43}$ East Anglian Regional Genetics Service, Cambridge University Hospitals NHS Foundation Trust, Addenbrooke's Hospital, Cambridge, United Kingdom. ${ }^{44}$ Manchester Centre for Genomic Medicine, Division of Evolution and Genomic Sciences, St Mary's Hospital, Manchester University Hospitals NHS Foundation Trust Manchester Academic Health Sciences Centre, Manchester, United Kingdom. ${ }^{45}$ Center for Human Genetics Inc, Cambridge, MA, USA. ${ }^{46}$ Department of Pediatrics, Central Hospital, Aichi Human Service Center, Kasugai, Aichi, Japan. ${ }^{47}$ Department of Pediatrics, Geisel School of Medicine, Dartmouth College, Hanover, NH, USA. ${ }^{48}$ Institute of Human Genetics, University Hospital of Cologne, Cologne, Germany.

${ }^{49}$ Gemeinschaftspraxis für Humangenetik Homburg/Saar, Homburg, Germany. ${ }^{50}$ Department of Medical Genetics, Osaka Women's and Children's Hospital, Osaka, Japan. ${ }^{51}$ Willem Alexander Children's Hospital, Leiden University Medical Center, Leiden, The Netherlands. ${ }^{52}$ CRMR Déficiences intellectuelles, Service de Génétique Médicale, CLAD Ouest CHU Hôpital Sud, Rennes, France. ${ }^{53}$ All Wales Medical Genetics Service, Glan Clwyd Hospital, Rhyl, United Kingdom. ${ }^{54}$ Dunedin School of Medicine, University of Otago, Dunedin, New Zealand. ${ }^{55}$ Division of Clinical and Metabolic Genetics, Department of Paediatrics, The Hospital for Sick Children, University of Toronto, Toronto, ON, Canada. ${ }^{56}$ The Prenatal Diagnosis and Medical Genetics Program, Department of Obstetrics and Gynecology, Mount Sinai Hospital, Toronto, ON, Canada. ${ }^{57}$ Department of Medical Genetics, CHU Bordeaux, Bordeaux, France. ${ }^{58}$ Department of Pediatrics, Leiden University Medical Center, Leiden, The Netherlands. ${ }^{59}$ Genpute Computation Technologies Company, Istanbul, Turkey. ${ }^{60}$ Department of Clinical Genetics and GROW-School for Oncology and Developmental Biology, Maastricht University Medical Center, Maastricht, The Netherlands. ${ }^{61}$ Servicio de Pediatría, Hospital Universitario Doctor Peset, Valencia, Spain. ${ }^{62}$ Department of Pediatric Genetics, Ihsan Dogramaci Children's Hospital, Hacettepe University School of Medicine, Ankara, Turkey. ${ }^{63}$ Jacinto de Magalhães Medical Genetics Center, Centro Hospitalar do Porto, Porto, Portugal. ${ }^{64}$ Departamento de neurometabólicas, Hospital Universitario Son Espases, Palma de Mallorca, Spain. ${ }^{65} \mathrm{Karadeniz} \mathrm{Technical}$ University, Faculty of Medicine, Dept of Child Neurology, Retired Professor, Trabzon, Turkey. ${ }^{66}$ Northern Genetics Service, Institute of Genetics Medicine, Newcastle upon Tyne, United Kingdom. ${ }^{67}$ Division of Pediatrics, Yamagata Prefectural and Sakata Munici pal Hospital Organization Nihon-Kai General Hospital, Sakata, Japan. ${ }^{68}$ Enva Engineering, Ankara, Turkey. ${ }^{69}$ Division of Neurology, Department of Pediatrics, Children's Hospital of Eastern Ontario, University of Ottawa, Ottawa, ON, Canada. ${ }^{70}$ Department of Genetics, Hôpital Universitaire des Enfants Reine Fabiola, ULB Center of Medical Genetics, Université Libre de Bruxelles, Brussels, Belgium. ${ }^{71}$ Department of Genetics, Hôpital Erasme. ULB Center of Medical Genetics, Université Libre de Bruxelles, Brussels, Belgium. ${ }^{72}$ Service de génétique clinique Guy Fontaine, CHRU de Lille-Hôpital Jeanne de Flandre, Lille, France. ${ }^{73}$ Division of Genetics, Arnold Palmer Hospital, Orlando, FL, USA. ${ }^{74}$ KinderGenome Genetics, Medical City Hospital Dallas, Dallas, TX, USA. ${ }^{75}$ Institute of Human Genetics, University Medical Center Göttingen, Göttingen, Germany. ${ }^{76}$ Center for Medical Genetics, Shinshu University Hospital, Matsumoto, Japan. ${ }^{77}$ Institute of Human Genetics, Medical Faculty, Heinrich-Heine-University, Düsseldorf, Germany. ${ }^{78}$ Department of Genome Sciences, University of Washington School of Medicine, Seattle, WA, USA 